\title{
MODEL ENDGAME ANALYSIS
}

\author{
G.M ${ }^{\mathrm{c} C}$. Haworth, R.B. Andrist \\ guy_haworth@hotmail.com; rba_schach@gmx.ch
}

\begin{abstract}
A reference model of Fallible Endgame Play has been implemented and exercised with the chess engine WILHELM. Various experiments have demonstrated the value of the model and the robustness of decisions based on it. Experimental results have also been compared with the theoretical predictions of a Markov model of the endgame and found to be in close agreement.
\end{abstract}

Keywords: chess, endgame, experiment, fallibility, Markov, model, theory

\section{Introduction}

In Haworth (2003), a reference model of fallible endgame play was defined in terms of a spectrum of Reference Endgame Players (REPs) $R_{c}$. The REPs are defined as choosing their moves stochastically, using only successor positions' values and depths from an endgame table (EGT). Exploring here the parameters of the model and various opponent-sensitive uses of the REPs including choice of move, we report on:

a) the robustness of decisions based on the model,

given that various parameters of the model may be changed,

b) the apparent competence of reference player $R_{20}$ in an $R_{20}-R_{\infty}$ match,

c) the distribution of game lengths in that match versus Markov theory,

d) the probability of beating a 50-move draw claim versus Markov theory,

e) the apparent competence of carbon and silicon players over the board.

In Section 2, we revisit the basic concepts and theory of the REP model, while in Section 3, we describe the REP implementation in WILHELM (Andrist, 2003). In Sections 4 to 7, we focus on the five topics above. Section 8 summarises and notes some questions arising from this work. 


\section{The Reference Endgame Player Model}

A nominated endgame, e.g., chess' $\mathrm{KQKR}$, is considered to be a system with a finite set of states $\left\{s_{i}\right\}$ numbered from 0 to $n s-1 .{ }^{1}$ Each state $s(v a l, d)$ is an equivalence class of positions of the same theoretical value val and depth $d$. Higher-numbered states are assumed to be less attractive to the side to move, which is taken to be White. Thus, for $\mathrm{KQKR}$ with the DTC ${ }^{2}$ metric, we have maxDTCs $(1-0) n_{w}=31,(0-1) n_{B}=3$, and $n s=37$ states in total:

- $s_{i}, i=0$ : a 1-0 win, i.e., for White, not requiring a winner's move ${ }^{3}$,

- $s_{i}, 1 \leq i \leq 31: 1-0$ wins of depth $i$,

- $s_{i}, i=32$ : theoretical draw, either in the endgame or a subgame,

- $s_{i}, 33 \leq i \leq 35$ : 0-1 wins, i.e., for Black, of depth 36-i

- $s_{i}, i=36$ : a $0-1$ win not requiring a winner's move.

The REP $R_{c}$ in position $P$ chooses stochastically from moves which each have a probability proportional to a Preference ${ }^{4}, S_{c}\left(v a l_{s}, d_{s}\right)$, where $s$ is the move's destination state with theoretical value $v a l_{s}$ and win/loss depth $d_{s}$. Each move-choice by $R_{c}$ is independent of previous move-choices.

We require that $\left\{R_{c}\right\}$ is a spectrum of players, ranging linearly from the metric-infallible player $R_{\infty}$ via the random player $R_{0}$ to the anti-infallible player $R_{-\infty}$. To ensure this, the function $S_{c}(v a l, d)$ is required to meet some natural criteria, as described more fully and formally in Haworth (2003) and in Appendix B.

Here, we choose, as an $S_{c}(v a l, d)$ function meeting those criteria:

$S_{c}($ win,$d) \equiv(d+\kappa)^{-c}$ with $\kappa>0$ to ensure that $S_{c}$ is finite,

$S_{c}($ draw $) \equiv S_{c}$ (win, $\left.n_{l}\right) \equiv S_{c}$ (loss, $\left.n_{2}\right)$ with $n_{1}>n_{W}$ and $n_{2}>n_{B}$,

$S_{c}($ loss,$d) \equiv \lambda \cdot(d+\kappa)^{c}, \lambda$ being defined by $n_{1}$ and $n_{2}$ above.

This ensures, as required, that $R_{0}$ prefers no move to any other, that $R_{c}$ with $c>0$ prefers better moves to worse moves, and that as $c \rightarrow \infty$, the $R_{c}$ increase in competence and tend to infallibility in terms of the chosen metric.

Although the $R_{c}$ have no game-specific knowledge, the general REP model allows moves to be given a prior, ancillary, weighting $v_{m}$ based on such considerations (Jansen, 1992). Thus, $v_{m}=0$, as used in this paper, prevents a move being chosen and $v_{m}>1$ makes it more likely to be chosen.

The probability $T_{c}(i)$ of moving to state $s_{i}$ is therefore:

$$
T_{c}(i) \equiv S_{c}\left(s_{i}\right) \cdot \sum_{\text {moves_to_state_ } i} v_{m} / \sum_{\text {all_moves }} v_{m} \cdot S_{c}\left(s_{\text {move }}\right)
$$

\footnotetext{
${ }^{1}$ For convenience, Appendix A summarises the key acronyms, notation, and terms.

${ }^{2}$ DTC $\equiv$ DTC (onversion) $\equiv$ Depth to Conversion, i.e., to mate and/or change of material.

${ }^{3}$ i.e., mate, achieved conversion to won subgame, or loser forced to convert on next move.

${ }^{4}$ For convenience and clarity, the Preference Function $S_{c}\left(v a l_{s}, d_{s}\right)$ may be signified by the more compact notations $S_{c}(v a l, d)$ or merely $S_{c}(s)$ if the context allows.
} 


\section{Implementing the REP Model}

The second author has implemented in WILHELM (Andrist, 2003) a subset of the REP model which is sufficient to provide the results of this paper.

Ancillary weightings $v_{m}$ are restricted to 1 and $0 . v_{m}=0$ is, if relevant, applied to all moves to a state $s$ rather than to specific moves: it can be used to exclude moves losing theoretical value, and/or to emulate a search horizon of $H$ moves, within which a player will win or not lose if possible.

WILHELM offers five agents based on the REP model: these are, as defined below, the Player, Analyser, Predator, Emulator, and Predictor. A predefined number of games may be played between any two of WILHELM, Player, Predator, Emulator and an infallible player with endgame data. WILHELM also supports the creation of Markov matrices, see Section 5.

\subsection{The Player}

The Player is an REP $R_{c}$ of competence $c$, and therefore chooses its moves stochastically using a validated (pseudo-)random number generator in conjunction with the function $S_{c}(v a l, d)$ defined earlier.

\subsection{The Analyser}

Let us imagine that an unknown fallible opponent is actually going to play as an $R_{c}$ with probability $p(d) \cdot \delta x$ that $c \in(x, x+\delta x): \int p(x) \mathrm{dx}=1$.

The Analyser attempts to identify the actual, underlying $c$ of the $R_{c}$ which it observes. For computational reasons, the Analyser must assume that $c$ is a value from a finite set $\left\{c_{j}\right\}$ and that $c=c_{j}$ with initial probability $p c_{0, j}$.

Here, the $c_{j}$ are regularly spaced in $\left[c_{\min }, c_{\max }\right]$ as follows:

$$
c_{\min }=c_{1}, c_{j}=c_{1}+(j-1) \cdot c \delta \text { and } c_{\max }=c_{1}+(n-1) \cdot c \delta \text {, i.e. } c=c_{\min }(c \delta) c_{\max } \text {. }
$$

The notation $c=c_{\min }(c \delta) c_{\max }$ is used to denote this set of possible values $c$. The initial probabilities $p c_{0, j}$ may be $1 / n$, the usual 'know nothing' uniform distribution, or may be based on previous experience or hypothesis. They are modified, given a move to state $s_{\text {next }}$, by Bayesian inference:

$T_{j}($ next $)=\operatorname{Prob}\left[\right.$ move to state $s_{\text {next }} \mid c=c_{j}$, and

$p c_{i+1, j}=p c_{i, j} T_{j}($ next $) / \sum_{k}\left[p c_{i, k} \cdot T_{k}(\right.$ next $\left.)\right]$.

Thus, the new Expected $[c]=\sum_{j} p c_{i+1, j} \cdot c_{j}$.

In Subsection 4.1, we investigate what values should be chosen for the parameters $c_{\min }, c \delta$ and $c_{\max }$ so that the errors of discrete approximation are acceptably small. 


\subsection{The Predator}

On the basis of what the Predator has learned from the Analyser about its opponent, it chooses its move to best challenge the opponent, i.e., to optimise the expected value and depth of the position after a sequence of moves. As winning attacker, it seeks to minimise expected depth; as losing defender, it seeks to maximise expected depth. In a draw situation, it seeks to finesse a win.

Different moves by the Predator create different sets of move-choices for the fallible opponent. These in turn lead to different expectations of theoretical value and depth after the opponent's moves.

The predator implementation in WILHELM chooses its move on the basis of only a 2-ply search. It may be that deeper searches will be worthwhile, particularly in the draw situation.

\subsection{The Emulator}

The Emulator $E_{c}$ is conceived as a practice opponent with a 'designer' level of competence tailorable to the requirements of the practising player. An REP $R_{c}$ will exhibit an apparent competence $c^{\prime}$ varying, perhaps widely, above and below $c$ because it chooses its moves stochastically. In contrast, the Emulator $E_{c}$ chooses a move which exhibits to an Analyser an apparent competence $c^{\prime \prime}$ as close to $c$ as possible.

The reference Analyser is defined as initially assuming the Emulator is an $R_{x}, x=0(1) 2 c$, where $x=x_{j}$ with initial probability $1 /(2 c+1)$.

The Emulator $E_{c}$ therefore opposes a practising player with a more consistent competence $c$ than would $R_{c}$, albeit with some loss of variety in its choice of moves. The value $c$ can be chosen to provide a suitable challenge in the practice session.

The practising player may also have their apparent competence assessed by the Analyser.

\subsection{The Predictor}

The Predictor is advised of the apparent competence $c$ of the opponent. It then predicts how long it will take to win, or what its chances are of turning a draw into a win, using data from an Analyser and from a Markov model of the endgame. This model is defined in Section 5. 


\section{Robustness of the Model}

The two famous Browne-BELLE KQKR exhibition games have already been studied using the REP model (Haworth, 2003). Browne's apparent competence $c$ was assessed by an Analyser, and BELLE's moves as Black were compared with the decisions of a Predator using the Analyser's output.

In that analysis, the following six choices were made:

- $c_{\min }=0, c \delta=1, c_{\max }=50$; all $c_{j}$ were deemed equally likely,

- $\quad \kappa=0^{+}$(i.e., arbitrarily small, effectively zero) and metric $=$DTC.

The following question therefore arises: to what extent are the conclusions of the Analyser and the choices made by the Predator affected by these six choices? Our first studies addressed this question.

\subsection{The Effect of Numerical Approximation}

Browne-BELLE game 1 was first reanalysed, this time with $\kappa=1$, and:

$c_{\min }=0, c_{\max }=50$ and $c \delta$ in turn set to $0.01,0.1,1,2,5$ and 10 .

Figure 1 takes the Analyser with $c \delta=0.01$ as a benchmark, and shows how the choice of $c \delta$ affected the Analyser's inferences during play.

It may be shown the Analyser's Bayesian calculation is a discrete approximation to a calculable integral: the theory of integration therefore guarantees that this calculation will converge as $c \delta \rightarrow 0$. We judge that the error is ignorable with $c \delta=1$ and that no smaller $c \delta$ is needed.

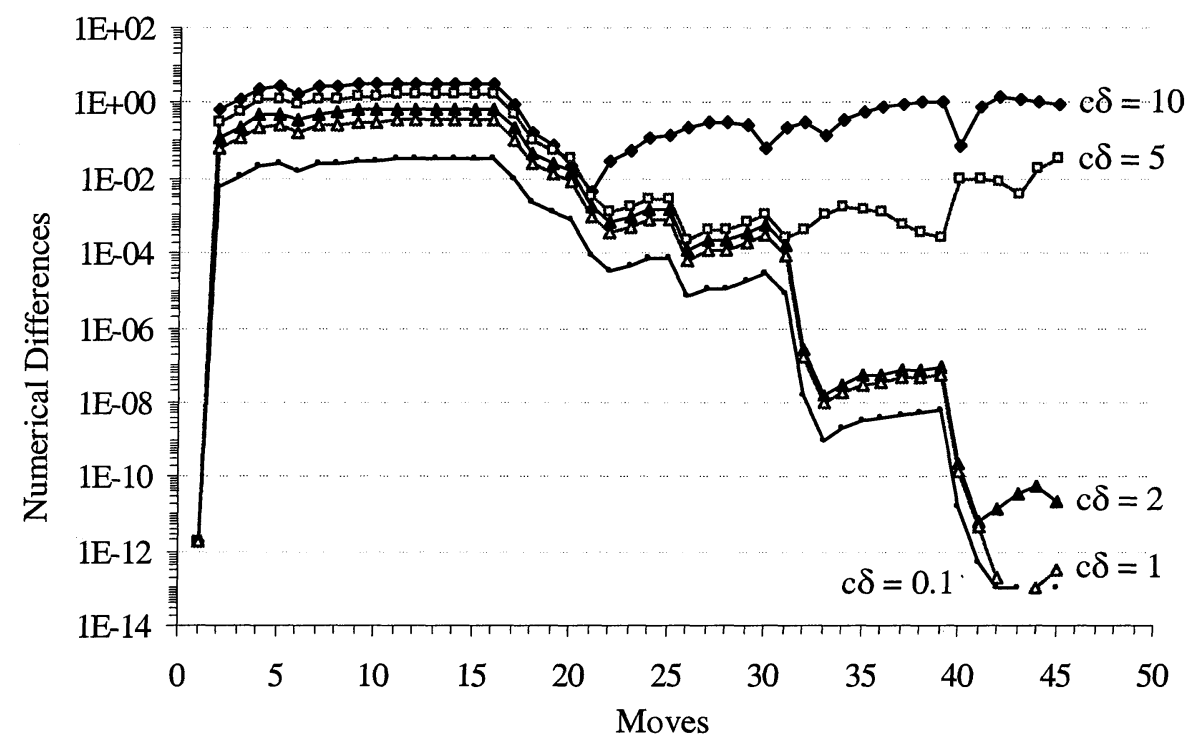

Figure 1. Differences in $c$-estimation, relative to the $c \delta=0.01$ estimate. 


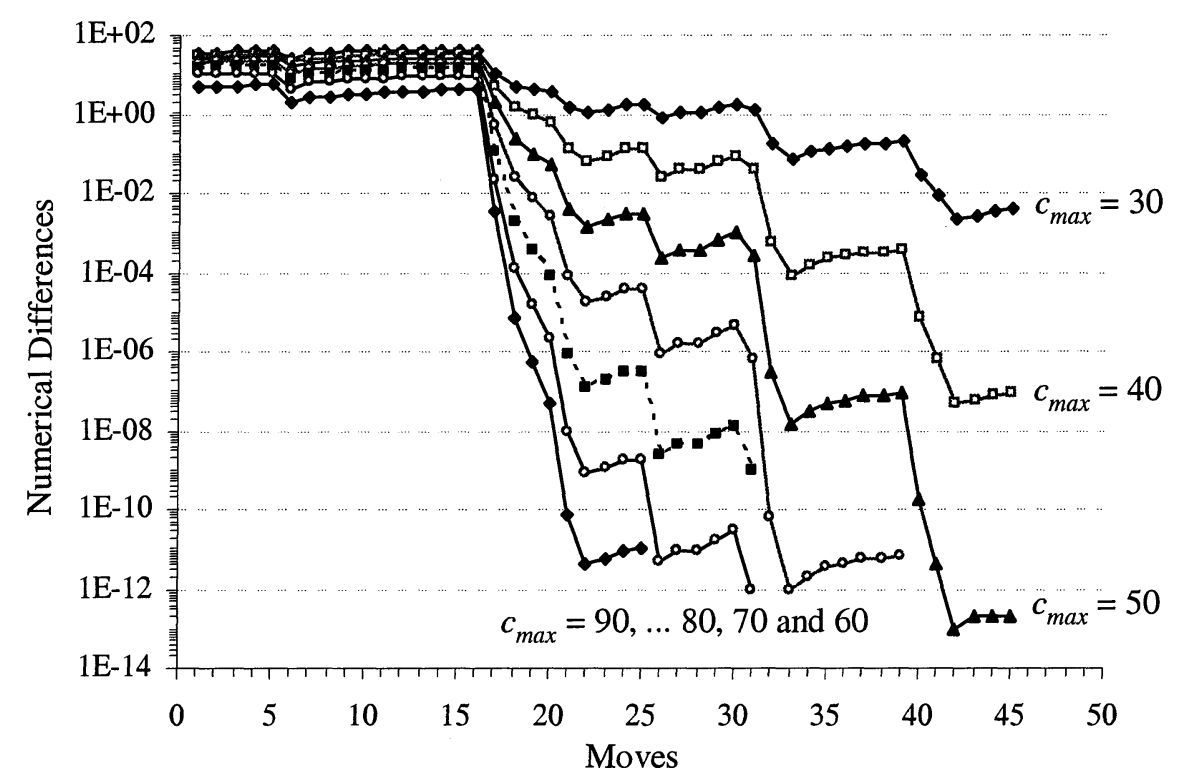

Figure 2. Differences in $c$-estimation, relative to the $c_{\max }=100$ estimate.

The analysis of the game was then repeated with:

$c \delta=1, c_{\min }=0$ and $c_{\max }$ in turn set to $100,90,80,70,60,50,40$ and 30 .

Again, intuitively, we would expect the error introduced by a finite $c_{\max }$ to reduce as $c_{\max } \rightarrow \infty$. Figure 2 shows that this is indeed the case and that, with Browne's apparent $c \approx 20, c_{\max }=50$ is conservative enough. However, it may need to be larger for easier endgames.

We assume that our opponent has positive apparent competence $c$ and that the Analyser is correct in taking $c_{\min }=0$ as a lower bound on $c$.

\subsection{The Effect of $k$}

Given the requirements on $S_{c}(v a l, d)$, it may be shown ${ }^{5}$ that, as $\mathrm{K}$ increases, $R_{c}$ progressively loses its ability to differentiate between better and worse moves, that $R_{c}$ 's expectation of state and theoretical value do not improve and that $R_{c} \rightarrow R_{0}$. Thus, for a given set of observations, if the Analyser assumes a greater $\kappa$, it will infer an increasing apparent competence $c$.

In this paper, we choose a fixed $\kappa=1$ throughout, as it were, recognising the next move in the line contemplated. We have not tested the effect of different $\kappa$ on a Predator's choices of move, but assume it is not great. There seems little reason to choose one value of $\kappa$ over another.

\footnotetext{
${ }^{5}$ The proof is by elementary algebra and in the style of Theorem 3 (Haworth, 2003).
} 


\subsection{The Effect of the Initial Probability Assumption}

The usual, neutral, initial stance is a know nothing one, assuming that $c$ is uniformly distributed in a conservatively-wide interval $\left[c_{\min }, c_{\max }\right]$. However, it is clear that had BELLE been using the REP model, it could have started game two with its perception of Browne as learned from game one, just as Browne started that game with his revised perception of KQKR. Also, one might have a perception of the competence $c$ likely to be demonstrated by the opponent with the given endgame force - and choose this to be the midpoint of a $\left[c_{\min }, c_{\max }\right]$ range with a normal distribution.

Bayesian theory, see Subsection 3.2, shows that the initial, assumed nonzero probabilities continue to appear explicitly in the calculation of subsequent, inferred probabilities. We therefore conclude that initial probabilities have some effect on the inferred probabilities.

\subsection{The Effect of the Chosen Metric}

The metric Depth to Conversion (DTC) was chosen because conversion is an obvious intermediate goal in most positions. The adoption of DTC is however a chessic decision.

Our analysis of the Browne-BELLE games shows that the Predator would never have made a DTC-suboptimal move-choice for Black. It is reasonable to assume that, had DTM(ate) been the chosen metric, it would never have chosen a DTM-suboptimal move.

However, different metrics occasionally define different subsets of moves as metric-optimal. Where this occurs, the Predator might well choose a different move in its tracking of the Browne-BELLE games.

\section{A Markov Model of the Endgame}

Let us assume that the Preference Function $S_{c}(v a l, d)$ is fixed, e.g., as the function defined here with $\kappa=1$.

Given a position $P$ in state $s_{i}$, we can calculate the probability of $R_{c}$ choosing move $m$ to some position $P^{\prime}$ in state $s_{j}$. We may therefore calculate the probability, $T_{c}(j)$ of moving from position $P$ to state $s_{j}$. Averaging this across the endgame over all such positions $P$ in state $s_{i}$, we may derive the probability $m_{i, j}$ of a state transition $s_{i} \rightarrow s_{j}$ assuming initial state $s_{i}$.

The $\left\{m_{i, j}\right\}$ define a Markov matrix $\mathbf{M}_{\mathbf{c}}=\left[m_{i, j}\right]$ for player $R_{c}$. This matrix, and the predictions which may be derived from it, provide a characterisation of the endgame as a whole.

Let us assume that the initial position is $1-0$, in state $s_{i}$, and that $R_{c}$ does not concede the win. From the matrix, we may derive predictions such as: 
- the probability of $R_{c}$ winning on or before move $m$,

- the expected number of moves required for $R_{c}$ to achieve the win.

this is $L_{i}$ in the solution of $\left(\underline{\mathbf{I}}-\mathbf{M}_{\mathbf{c}}\right) \cdot \underline{\mathbf{L}}=\underline{\mathbf{U}}^{6}$, q.v. (Haworth, 2003).

These theoretical predictions have been computed and are compared with the results of the extensive experiment described in the next section.

\section{An Experiment with $\mathbf{R}_{20}$}

Echoing Browne-BELLE, a model KQKR match was staged between the fallible attacker $R_{20}$ and the infallible defender $R_{\infty}$. It was assumed that $R_{20}$ would not concede the win but eventually secure it as theory predicts. The game-specific repetition and 50-move drawing rules were assumed not to be in force. Table 1 summarises the results of this experiment.

1,000 games were played from each of the two maxDTC KQKR positions used in the Browne-BELLE match. Games ended when conversion was achieved by White. The purpose of the experiment was to observe:

- the distribution of the $c$ inferred by an Analyser ${ }^{7}$ at the end of each game with the assumed probability of $c_{i}$ set to $1 / 51$ at start of each game,

- the distribution of the lengths of the games, and

- the trend in the Analyser's inferred $c$, ignoring game-starts after the first.

\begin{tabular}{lccc}
\hline KQKR: $\mathbf{R}_{\mathbf{2 0}}-\mathbf{R}_{\boldsymbol{\infty}}$ & Position 1 & Position 2 & Overall \\
Min., end-of-game apparent $c$ & 15.06 & 14.73 & 14.73 \\
Max., end-of-game apparent $c$ & 35.66 & 40.71 & 40.71 \\
Mean, end-of-game apparent $c$ & 21.318 & 21.620 & 21.469 \\
St. Dev., end-of-game apparent $c$ & 3.345 & 3.695 & 3.524 \\
St. Dev of the Mean apparent $c$ & 0.106 & 0.117 & 0.079 \\
|Mean $c$ - 20|/Stdev_mean & 12.43 & 13.85 & 18.59 \\
Min. moves, $m$, to conversion & 37 & 37 & 37 \\
Maximum moves, $m$ & 395 & 325 & 395 \\
Mean moves, $m$ & 96.88 & 94.31 & 95.60 \\
St. Dev., $m$ & 102.951 & 102.273 & 102.587 \\
St. Dev., mean of $m$ & 3.256 & 3.234 & 2.294 \\
\hline
\end{tabular}

Table 1. Statistical Analysis of the 2,000-game experiment.

${ }^{6} \mathbf{I}$ is the Identity matrix; $\underline{\mathbf{U}}$ is a vector where each element is the unit ' 1 '.

${ }^{7}$ using $c_{\min }=0, c \delta=1$ and $c_{\max }=50$ as found adequate in Section 4.1. 


\section{1 $R_{20}$ 's Apparent c after One Game}

Figure 3 shows the distribution of the apparent $c$ as inferred at the end of each, single game: the mean $c$ is $21.50 \pm 0.08^{8}$. This rather surprised us, being more distant from the actual $c=20$ than expected. The reason is that the mean of $\{$ end-of-game estimated $c\}$ is not statistically the best way to estimate the underlying $c$, a task we revisit in Subsection 6.4.

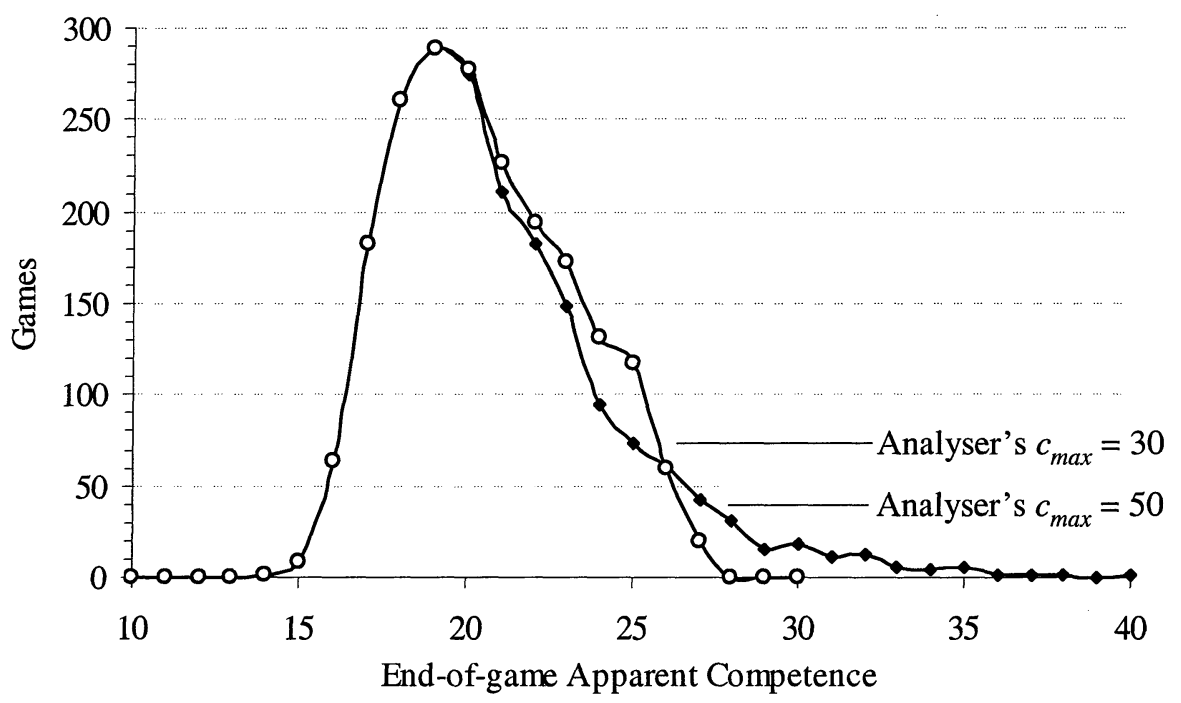

Figure 3. Distribution of apparent $c$ as inferred after one game.

\subsection{Game-Length Statistics}

Starting from the two positions with (maximum) DTC depth 31 , and taken over the 2,000 games, the mean number of moves required for conversion is $95.60 \pm 2.29$. Figure 4 shows the distribution of the experiment's game lengths in comparison with the predictions of the Markov model.

Figure 5 shows the Markov-model predictions for the expected number of moves to conversion, for $c=20,21$, and 22 and starting at any depth. Note that it shows that the main barriers to progress seem to be between depths 17 and 26 rather than at the greatest depths.

From depth 31, the moves predicted are 97.20 for $\mathrm{c}=20,83.70$ for $\mathrm{c}=21$ and 74.16 for $\mathrm{c}=22$. The experimental results are therefore in close agreement with these predictions, indicating a $c$ of $\sim 20.1$.

\footnotetext{
${ }^{8}$ Mean end-of-game apparent $c$ is still 21.04 when the Analyser' $c_{\max }$ is 30 rather than 50 .
} 


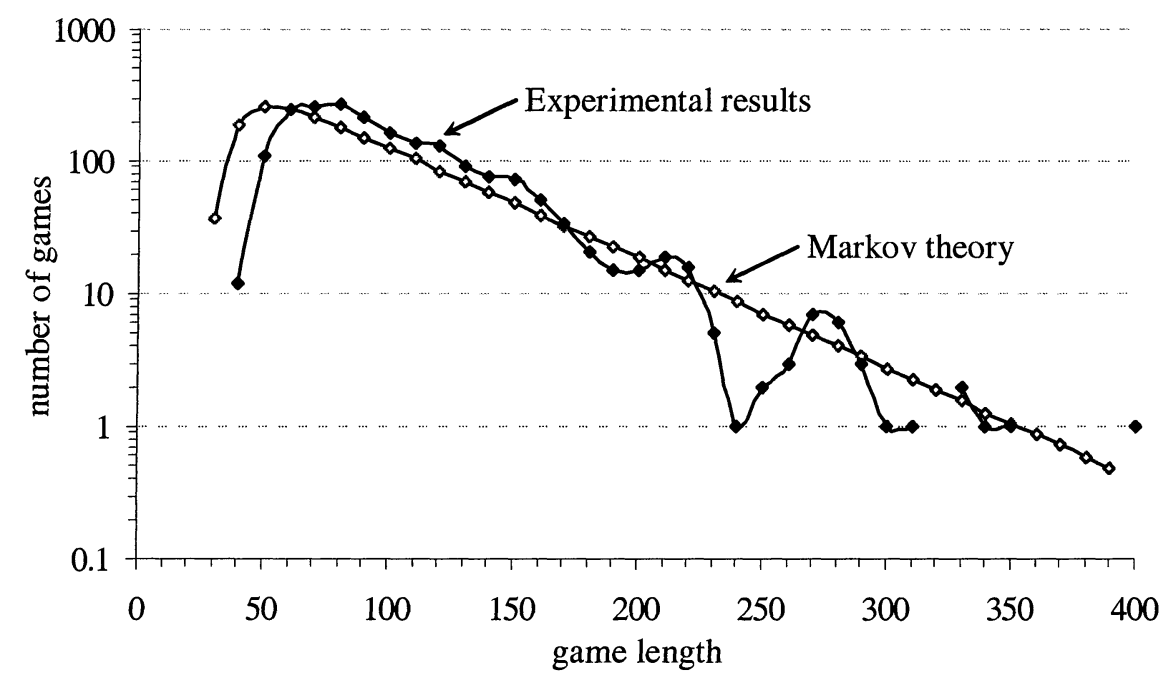

Figure 4. Distribution of game lengths in the $R_{c}-R_{\infty} \mathrm{KQKR}$ match.

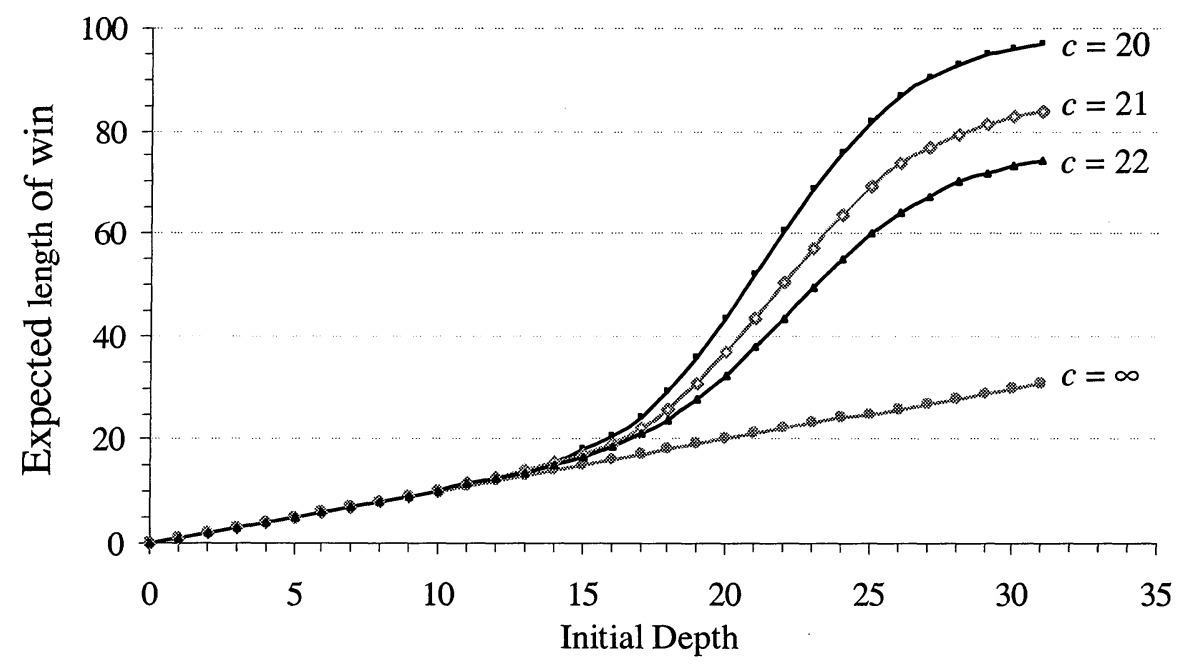

Figure 5. Expected[moves to conversion in an $R_{c}-R_{\infty} \mathrm{KQKR}$ game].

\subsection{The Probability of Winning}

The games were played without the 50-move rule but the Markov model allows us to calculate the probability of winning from depth $d$ on or before move 50 , before a possible draw claim by the opponent. It is the probability of being in state 0 after 50 moves, namely the element $\mathbf{M}_{\mathbf{c}}{ }^{50}[d, 0]$ of $\mathbf{M}_{\mathbf{c}}{ }^{50}$. 
Figure 6 gives these probabilities for $c=20,21$, and 22 and for all initial depths. For $c=20$ and initial depth 31 , this is $12.67 \%$, a figure reached after 55 moves in the 2,000 game experiment.

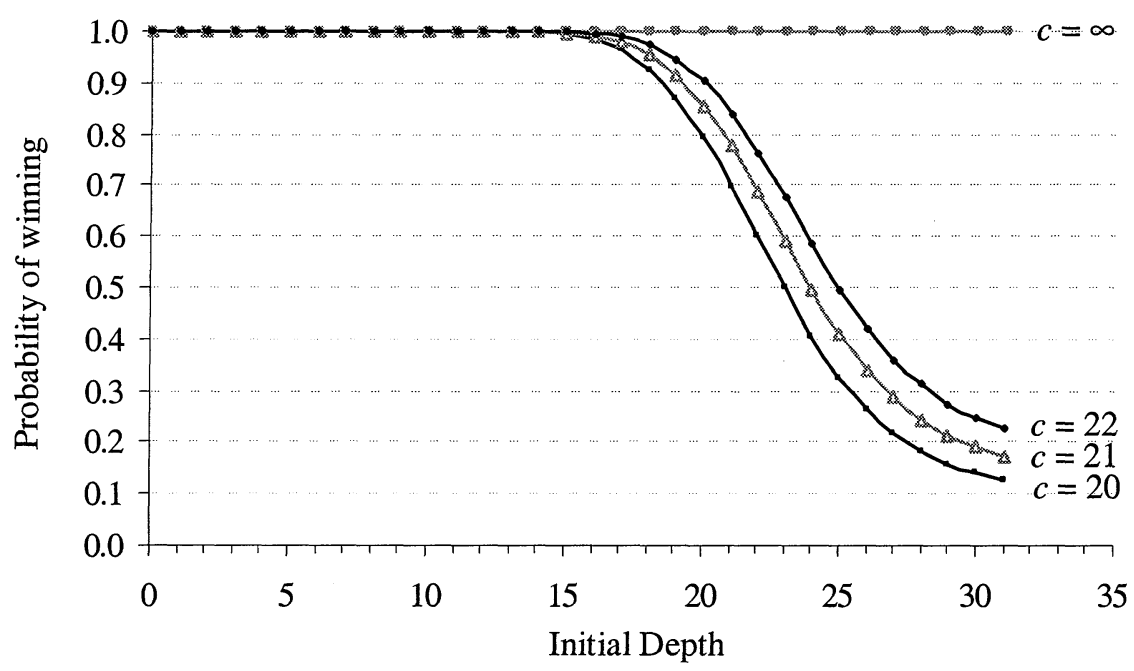

Figure 6. Probability[ $R_{c}$ wins an $R_{c}-R_{\infty} \mathrm{KQKR}$ game in 50 moves].

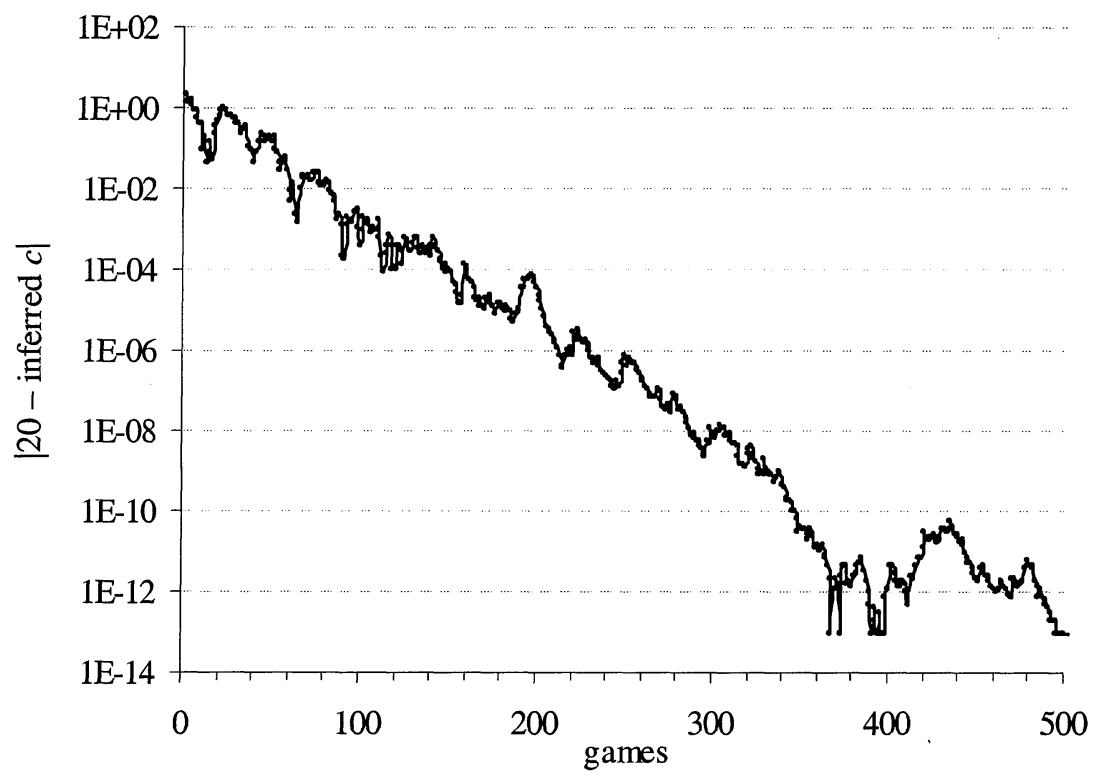

Figure 7. Analyser error in $c$-estimate versus number of games analysed. 


\subsection{Analysing $R_{c}$ 's competence $c$}

The mean of the 2,000 end-of-game apparent $c$ values is not actually the best estimate of $R_{c}$ 's underlying $c$.

The reason is that the 2,000 games may be seen as some 191,200 independent move-choices by the $R_{c}$. There is no need to associate the know nothing uniform distribution probabilities with the possible $c_{j}$ more than once. In fact, to do so is to interrupt the Bayesian inference processor of the Analyser and to negate what the Analyser has learned from previous games about the non-uniform distribution of probabilities of the candidate $c_{j}$.

Figure 7 shows the Analyser's perception of $c$ approaching the correct value of 20 as it works through the 2,000 games. Even starting with an estimate of $c=25$, it is accurate to 0.1 after examining 6,000 moves.

\section{Apparent Competence of Players}

The apparent competence of both carbon and silicon players has been calculated for some published games. The initial assumptions differed slightly from those of Haworth (2003): here, WILHELM was set to analyse with candidate $c=0(0.01) 50$ and $\kappa=1$ and the results are listed in Table 2, showing depth conceded by both sides, net progress and apparent $c$.

Some background to the games may help put the figures in context. The two Browne-BELLE games (Fenner, 1979; Haworth, 2003; Jansen, 1992a; Levy and Newborn, 1991) are the famous demonstration that the 'easy' KQKR endgame is not so easy to win. Gelfand-Svidler was a tie-breaker rapid-play game played under extreme time pressure. Pinter-Bronstein has been extensively analysed by Roycroft (1988). Timman consulted extensively in a prior adjournment (Breuker et al, 1992). FRITZ (Heise, 2002) played itself with only 3-to-4-man EGTs in an Intel-AMD duel. Lengyel lost the draw three times before our analysis begins (Levy, 1972a,b, 1992).

\begin{tabular}{|c|c|c|c|c|c|c|c|c|c|c|c|}
\hline \multirow[b]{2}{*}{ \# } & \multirow[b]{2}{*}{ Profile } & \multirow[b]{2}{*}{ White } & \multirow[b]{2}{*}{ Black } & \multirow[b]{2}{*}{ Res. } & \multirow[b]{2}{*}{ Year } & \multirow[t]{2}{*}{$\# \mathbf{m}$} & \multicolumn{2}{|c|}{ depth lost } & \multirow{2}{*}{$\begin{array}{c}\text { depth } \\
\text { gain }\end{array}$} & \multicolumn{2}{|c|}{ Final $c$} \\
\hline & & & & & & & Wh. & Bl. & & Wh. & Bl. \\
\hline 1 & KQKR & Browne & BELLE 1 & $=$ & 1978 & 45 & 27 & 0 & 18 & 19.5 & $\infty$ \\
\hline 2 & KQKR & Browne & BELLE 2 & $1-0$ & 1978 & 50 & 19 & 0 & 31 & 18.4 & $\infty$ \\
\hline 3 & KRKQ & Gelfand & Svidler & $=$ & 2001 & 50 & 37 & 79 & 8 & 3.5 & 4.1 \\
\hline 4 & KNKBB & Pinter & Bronstein & $=$ & 1977 & 50 & 60 & 95 & 14 & 5.9 & 15.4 \\
\hline 5 & KBBKN & Popovich & Korchnoi & $=$ & 1984 & 31 & 74 & 45 & $i$ & 8.1 & 6.6 \\
\hline 6 & $\mathrm{KBBKN}$ & Timman & Speelman & $1-0$ & 1992 & 25 & 36 & 44 & 33 & 15.0 & 7.6 \\
\hline 7 & KNKBB & FRITZ & FRITZ & $=$ & 2002 & 49 & 169 & 210 & 8 & 2.9 & 3.2 \\
\hline 8 & KQKQN & Lengyel & Levy & $0-1$ & 1972 & 14 & 7 & 16 & 5 & 4.2 & 2.8 \\
\hline
\end{tabular}

Table 2. Apparent Competence of Players. 
Even after noting that the values $c$ are not necessarily the player's true equivalent $c$, and are meaningful only in relative rather than absolute terms, the performances of Browne and Timman stand out. FRITZ trades major depth with its clone-opponent and clearly misses the withheld perfect information. The time constraints of Rapid Play, and even third-phase 30'/game play in classical chess, mitigate against quality endgame play - arguably a loss to the world of chess.

\section{Summary}

We have examined the utility of a reference model of Fallible Endgame Play by both experiment and theory, using both a comprehensive REP implementation in WILHELM and Markov methods. Various demonstrations have shown opportunities for exploiting the model, and the robustness of decisions based on it. Experimental results have also been compared with the Markov predictions, with which they agree closely.

Experiments which remain to be carried out include:

- infallible White attacking fallible Black in a drawn position

e.g., in KBBKN, KNNKP, KNPKN, KQNKQ, KQPKP, or KRBKR,

- infallible Black pressing for a draw in a lost position

this requires additional EGT data on draws forced in $d$ moves,

- a more insightful Predator searching more than $2 p$ plies ahead, and

- use of the Emulator as a training partner for human players.

The REP model may be extended to other games where EGTs may be computed - to convergent games such as Chinese Chess, $8 \times 8$ checkers, International Draughts, and in principle if not in practice, to divergent placement games such as Hex and Othello.

If a search method can propose what it considers the best few moves in a position, each evaluated on an identical basis and therefore comparable, the concept of a stochastic player may be applied more generally than to just endgames for which perfect information is available.

\section{Acknowledgements}

We thank the ACG10 conference sponsors, organizers, and referees for their considerable support and advice on this paper. We thank Walter Browne for his excellent sporting example in facing up to Ken Thompson's silicon beast in the KQKR match of 1979. Also, we thank those who have generated and/or made available definitive EGT data over the years. 


\section{References}

Andrist, R. (2003). http://www.geocities.com/rba_schach2000/. WILHELM download.

Breuker, D.M., Allis L.V., Herik, H.J. van den, and Herschberg, I.S. (1992). A Database as a Second. ICCA Journal, Vol. 15, No. 1, pp. 28-39.

Fenner, C.J. (1979). Computer Chess, News about the North American Computer Chess Championship. The British Chess Magazine, Vol. 99, No. 5, pp. 193-200.

Haworth, G.M ${ }^{c}$ C. (2003). Reference Fallible Endgame Play. ICGA Journal, Vol. 26, No. 2, pp. 81-91.

Heise (2002). http://www.chessbase.de/heise/games/runde7.htm. Chess Dual Round 7: Fritz(Intel)-Fritz(AMD).

Jansen, P.J. (1992). KQKR: Awareness of a Fallible Opponent. ICCA Journal, Vol. 15, No. 3, pp. 111-131.

Levy, D.N.L. (1972a). Endgame analysis. CHESS, May.

Levy, D.N.L. (1972b). Endgame analysis. CHESS, August.

Levy, D.N.L. and Nefkens, H. (1992). Before Databases. ICCA Journal, Vol. 15, No. 1, pp. 40-42.

Levy, D. and Newborn, M. (1991). How Computers Play Chess, esp. pp. 144-148.

Roycroft, A.J. (1988). Expert against the Oracle. Machine Intelligence 11 (eds. J.E. Hayes, D. Michie and J. Richards) pp. 347-373. Oxford University Press, Oxford.

Tamplin, J. (2001). Private communication of some pawnless Nalimov-compatible DTC EGTs.

\section{Appendix A: Acronyms, Notation and Terms}

$\begin{array}{ll}\text { Analyser } & \text { an agent identifying a fallible opponent as an } R_{c} \text { player } \\ c & \text { the competence index of an REP } \\ c \delta & \text { the difference between adjacent } c_{i} \text { assumed by the Analyser } \\ c_{\text {max }} & \text { the maximum } c \text { assumed possible by the Analyser } \\ c_{\text {min }} & \text { the minimum } c \text { assumed possible by the Analyser } \\ d & \text { the depth (of win or loss) of a position in the chosen metric, e.g. DTC } \\ \text { DTC } & \text { Depth to Conversion, i.e. to change of material and/or mate } \\ \text { DTM } & \text { Depth to Mate } \\ \text { Emulator } & \text { an agent, } E_{c} \text {, choosing moves to best exhibit apparent competence } c \\ \text { Horizon } & \text { a search limit, within which } R_{c} \text { will win or not lose if possible } \\ \kappa & \kappa>0 \text { ensures that }(d+\kappa)^{-c} \text { is finite } \\ \lambda & \text { a scaling factor, matching the probability of loss to that of a draw } \\ L_{i} & \text { expected length of win (to conversion in winner's moves) from depth } i \\ \text { maxDTC } & \text { maximum DTC (depths) } \\ M_{c} & \left.\text { a Markov matrix [ } m_{i, j}\right] \\ m_{i, j} & \text { the probability, averaged over the endgame, that } R_{c} \text { in state } s_{i} \text { moves to } s_{j} \\ \text { metric } & \text { a measure of the depth of a position, usually in winner's moves } \\ n & \text { the number of different } c_{i} \text { assumed by an Analyser } \\ n_{1} & n_{1}>n_{W}, \text { ensures that draws are less preferable than wins } \\ n_{2} & n_{2}>n_{B}, \text { ensures that draws are more preferable than losses } \\ n_{B} & \text { the number of 'Black win' states } \\ n_{W} & \text { the number of 'White win' states } \\ n s & \text { the number of states for a chosen endgame and depth metric } \\ p(x) \cdot \delta x & \text { the probability that } R_{c} \text { 's } c \in[x, x+\delta x]\end{array}$




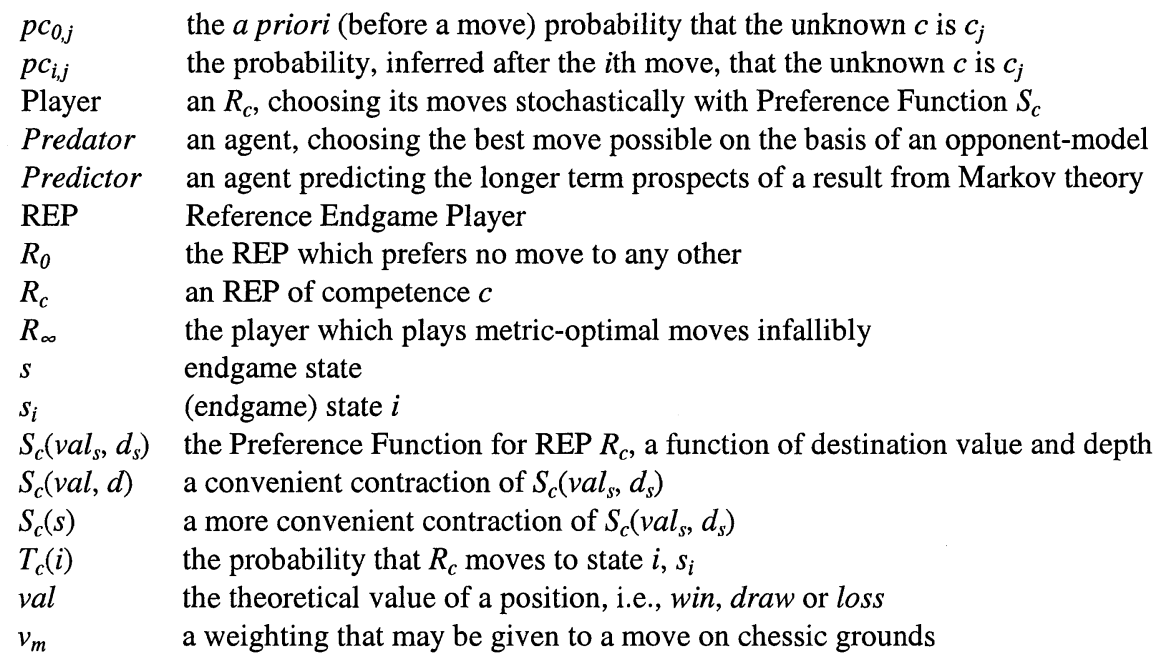

\section{Appendix B: Preference Functions}

We require that the set $\left\{R_{c}\right\}$ is in fact a linear, ordered spectrum of $R_{c}$ players such that:

- for $R_{0}$, all moves are equally likely,

- ' $R_{\infty}$ ' $\equiv \lim _{c \rightarrow \infty} R_{c}$ exists and is the infallible player choosing metric-optimal moves,

- ' $R_{-\infty}$ ' $\equiv \lim _{c \rightarrow-\infty} R_{c}$ exists and is the anti-infallible player choosing anti-optimal moves,

- $c 2>c 1 \Rightarrow R_{c 2}$ 's expectations of successor state, i.e. E[s], are no worse than $R_{c 1}$ 's,

- $c 2>c 1 \Rightarrow R_{c 2}$ 's expectations of theoretical value, i.e. E[ $\left.\mathrm{val}_{s}\right]$, are no worse than $R_{c 1}$ 's.

The following requirements on $S_{c}(v a l, d) \equiv S_{c}(s)$ are natural ones and sufficient to ensure the above, as proved in Haworth (2003):

$-S_{c}(s)$ is finite and positive: no move has zero or infinite preference for finite $c{ }^{9}$

- $S_{0}(s)$ is a constant,

- for some $n_{1}>n_{W}$ and $n_{2}>n_{B}, S_{c}($ draw $)=S_{c}\left(\right.$ win,$\left.n_{1}\right)=S_{c}\left(\right.$ loss, $\left.n_{2}\right)$,

$-F_{j}(c) \equiv S_{c}\left(s_{i+1}\right) / S_{c}\left(s_{i}\right)$ decreases as $c$ increases: $\lim _{c \rightarrow \infty} F_{j}(c)=0$ and $\lim _{c \rightarrow-\infty} 1 / F_{j}(c)=0$,

- for $c \neq 0$, $\operatorname{sign}(c) \cdot S_{c}\left(s_{j}\right)$ decreases $(\downarrow)$ as $j$ increases $(\uparrow)$,

- for $c>(<) 0, W_{c}(d)=S_{c}($ win, $d) / S_{c}($ win, $d+1) \downarrow(\uparrow)$ as $d \uparrow$ and $\lim _{d \rightarrow \infty} W_{c}(d)=1$,

- for $c>(<) 0, L_{c}(d)=S_{c}$ (loss, $\left.d+1\right) / S_{c}$ (loss, $\left.d\right) \downarrow(\uparrow)$ as $d \uparrow$ and $\lim _{d \rightarrow \infty} L_{c}(d)=1$.

The net effect is that:

- the spectrum of $R_{c}$ is centred as required on the random player, $R_{0}$,

- the $R_{c}$ with $c>0$ prefer better moves to worse moves,

- the $R_{c}$ demonstrate increasing apparent skill as $c \rightarrow \infty$,

- $R_{c}$ can be arbitrarily close to being the metric-infallible player for finite $c$

- as $d \rightarrow \infty, R_{c}$ discriminates less between a win (or loss) of depth $d$ and one of depth $d+1$.

${ }^{9}$ Hence the requirement that $\kappa>0$, to accommodate the case of $d=0$ in $(d+\kappa)^{-c}$. 